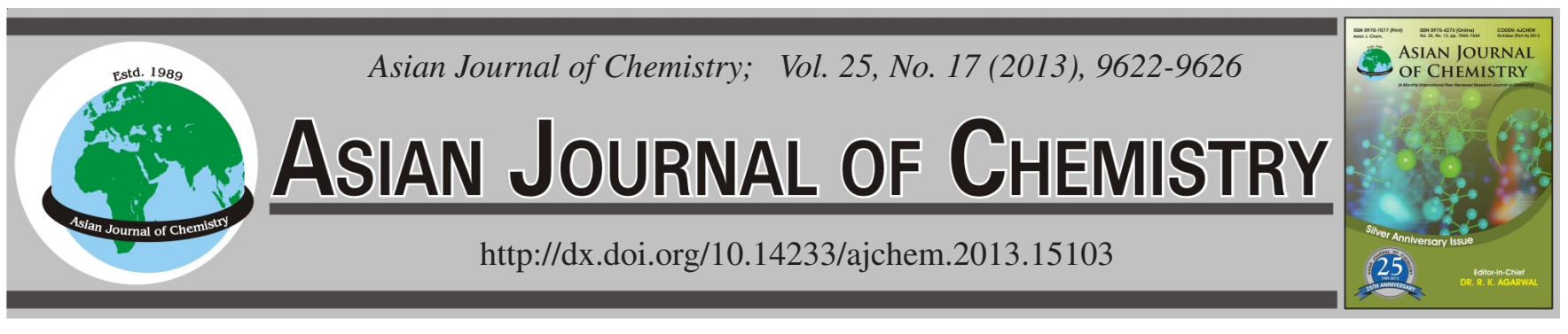

\title{
Functionalization of Carbon Microspheres Using 3-Methacryloxypropyl Trimethoxysilane and its Theoretical Elucidation
}

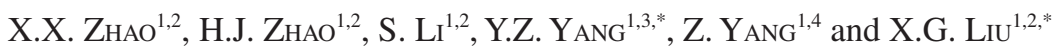 \\ ${ }^{1}$ Key Laboratory of Interface Science and Engineering in Advanced Materials (Taiyuan University of Technology), Ministry of Education, \\ Taiyuan 030024, P.R. China \\ ${ }^{2}$ College of Chemistry and Chemical Engineering, Taiyuan University of Technology, Taiyuan 030024, P.R. China \\ ${ }^{3}$ Research Center on Advanced Materials Science and Technology, Taiyuan University of Technology, Taiyuan 030024, P.R. China \\ ${ }^{4}$ College of Physical and Optoelectronics, Taiyuan University of Technology, Taiyuan 030024, P.R. China
}

*Corresponding authors: Tel./Fax: +86 351 6014138; E-mail: liuxuguang @tyut.edu.cn; yyztyut@ 126.com

(Received: 7 January 2013;

Accepted: 7 October 2013)

AJC-14241

\begin{abstract}
Carbon microspheres were oxidized by a mixture of concentrated sulfuric and nitric acids. Then oxidized carbon microspheres were modified by 3-methacryloxypropyl trimethoxysilane (KH-570) to prepare surface molecularly imprinted polymer for adsorbing dibenzothiophene. The effects of reaction time, KH-570 content and the ratio of ethanol to water on silanization were discussed. The morphologies and microstructures of all the samples were characterized by Fourier transform infrared spectrometry, field emission scanning electron microscopy, X-ray photoelectron spectroscopy and thermogravimetry. By virtue of the active sites on carbon microspheres, $\mathrm{KH}-570$ with the double bonds was grafted onto the surface of carbon microspheres, with the optimum parameters: $0.3 \mathrm{~g}$ of oxidized carbon microspheres, $1.0 \mathrm{~mL}$ of $\mathrm{KH}-570$, reaction for $2 \mathrm{~h}$ at $65^{\circ} \mathrm{C}, \mathrm{pH} \approx 5,60 \mathrm{~mL}$ of ethanol + water $(3: 1, \mathrm{v} / \mathrm{v})$. The dispersion of carbon microspheres in ethanol was also improved. The possible reaction mechanism was discussed on the basis of the density functional theory study. This lays an experimental and theoretical foundation for further application of functionalized carbon microspheres.
\end{abstract}

Key Words: Polymer, Simulation, Surface chemistry.

\section{INTRODUCTION}

Carbon microspheres (CMSs) are promising materials because of their large specific surface area, good mechanical and thermal stabilities ${ }^{1-7}$. However, their applications in many fields, such as bio-medicine, chemical industry and photoelectricity, have been limited by their inert chemical property and strong hydrophobicity. Therefore, it is an important project in the basic research field of CMSs to improve their hydrophilicity and dispersibility through surface modification. Recently, several methods, including gaseous or liquid-phase oxidation, polymer coating and plasma, were used for the surface treatment of carbon materials in order to develop their potential application $^{1-7}$. In grafting polymer monomers for surface imprinting, temperature response and polymer solar cells, CMSs are needed to have good dispersion and high activity for subsequent reactions in organic medium.

A coupling agent with bifunctional groups is a chemical function at the interface to create a chemical bridge between organic and inorganic materials. Silane coupling agents are regarded as efficient coupling agents with a generic chemical structure $R_{(4-n)}-\operatorname{Si}-\left(R^{\prime} X\right)_{n}(n=1,2)$, where $R$ is alkoxy, $X$ represents an organic functionality and $\mathrm{R}^{\prime}$ is an alkyl connecting the silicon atom and the organic functionality ${ }^{8}$.

In this paper, the surface of CMSs oxidized by a mixture of $\mathrm{HNO}_{3}$ and $\mathrm{H}_{2} \mathrm{SO}_{4}$ was modified by chemically grafting a coupling agent 3-methacryloxypropyl trimethoxysilane (KH570). The influences of reaction time, the content of $\mathrm{KH}-570$ and the ratio of ethanol to water were discussed. The $\mathrm{C}=\mathrm{C}$ bond was introduced onto the surface of CMSs by silanization, which is favorable for subsequent polymer grafting or coating on the surface of CMSs to fabricate polymer/CMSs core-shell structures and helpful for the realization of functionalized CMSs towards specialized applications, such as surface molecularly imprinted polymers (MIPs) for deep desulfurization of fuel oils ${ }^{1,9}$.

In order to get more information about the microscopic details of the chemical surface modification of CMSs, density functional theory (DFT) calculations were performed on the system. It is well known that DFT method is a powerful tool to investigate the electronic structures, chemical reactions and other physical or chemical properties of molecules or surfaces. All these provide a scientific basis for the application of CMSs 
in surface molecularly imprinted, environmentally responsive and photovoltaic materials ${ }^{1,9-11}$.

\section{EXPERIMENTAL}

Carbon microspheres with $350 \mathrm{~nm}$ in diameter were synthesized by chemical vapour deposition with $\mathrm{C}_{2} \mathrm{H}_{2}$ as carbon source and argon as carrier gas ${ }^{3}$. KH-570 ( $\geq 95$ wt \%) was obtained from Tianda Chemical Reagent Company, Tianjin, China. All other chemicals were of analytical grade and used as received. Distilled water was used to prepare all buffers and other solutions.

Oxidation of carbon microspheres: Firstly, the asprepared CMSs were treated in ethanol by ultrasonic vibration to obtain purified CMSs. To improve the surface activity of purified CMSs, oxygen-containing polar groups were introduced onto the surface of CMSs by oxidation treatment, i.e., by adding $1 \mathrm{~g}$ of CMSs to the mixed acids of $30 \mathrm{~mL}$ of $\mathrm{HNO}_{3}$ and $90 \mathrm{~mL}$ of $\mathrm{H}_{2} \mathrm{SO}_{4}$. The mixture was ultra-sonicated at $70{ }^{\circ} \mathrm{C}$ for $1 \mathrm{~h}$, filtered, washed with distilled water several times until the $\mathrm{pH}$ value approached to neutrality and then dried at $40^{\circ} \mathrm{C}$ in air to obtain oxidized-CMSs.

Silanization of oxidized-carbon microspheres: To further increase the reactivity of CMS surface, $\mathrm{C}=\mathrm{C}$ bond was introduced onto the surface of oxidized-CMSs by silanization with coupling agent KH-570, as shown in Scheme-I.<smiles>C=C(C)C(=O)CCC[Si](OC)(OC)OCCC(=O)C(C)=CCCO</smiles>

Scheme-I: Silanization of oxidized-CMSs by KH-570

A typical grafting process was as follows: oxidized-CMSs $(0.3 \mathrm{~g})$ were added to $60 \mathrm{~mL}$ of a mixed solvent of ethanol and distilled water $(\mathrm{v} / \mathrm{v}=3: 1)$, followed by the addition of $1.0 \mathrm{~mL}$ of KH-570. The mixture was refluxed in $\mathrm{N}_{2}$ atmosphere at 65 ${ }^{\circ} \mathrm{C}$ for $2 \mathrm{~h}$ under magnetic stirring. Then the samples were extracted with ethanol repeatedly to remove excess KH-570 and dried at $50{ }^{\circ} \mathrm{C}$ for $24 \mathrm{~h}$, resulting in the formation of silanized-CMSs. The influences of reaction time, KH-570 content and the ratio of ethanol to distilled water on silanization were investigated.

Detection method: The morphology and structure of the products were characterized by field emission scanning electron microscopy (FESEM, JSM-6700F, operated at $10 \mathrm{kV}$ ) with $\mathrm{X}$-ray energy dispersive spectroscopy (EDS). The functional groups were analyzed qualitatively by Fourier transform infrared spectrometry (FTIR). The grafting degree of KH-570 was investigated by thermogravimetry (TG, Netzsch 209 F3, between 100 and $800{ }^{\circ} \mathrm{C}$ in $\mathrm{N}_{2}$ atmosphere at heating rate of $10{ }^{\circ} \mathrm{C} / \mathrm{min}$ ).

The grafting percentage of CMSs with KH-570 was calculated according to eqn. 1 :
The grafting percentage of silanized-CMSs

$$
=\frac{\mathrm{W}_{\text {silanized-CMSs }}-\mathrm{W}_{\text {oxidized-CMSs }}}{\mathrm{W}_{\mathrm{KH}-570}-\mathrm{W}_{\text {oxidized-CMSs }}}
$$

where, $\mathrm{W}_{\text {oxidized-CMSs }}, \mathrm{W}_{\text {silanized-CMSs }}$ and $\mathrm{W}_{\mathrm{KH}-570}$ are weight loss of oxidized-CMSs, silanized-CMSs and KH-570, respectively.

\section{RESULTS AND DISCUSSION}

Oxidation of carbon microspheres: Fig. 1 shows the FESEM images of CMSs at different processing steps. It can be seen from Fig. 1(b) that purified-CMSs with few agglomeration had better dispersion than as-prepared CMSs [Fig. 1(a)], indicating that small molecules or carbon debris on the surface of the as-prepared CMSs were removed by ethanol purification. The oxidized-CMSs with uniform size were well dispersed in spherical shape with bare surfaces [Fig. 1(c)].
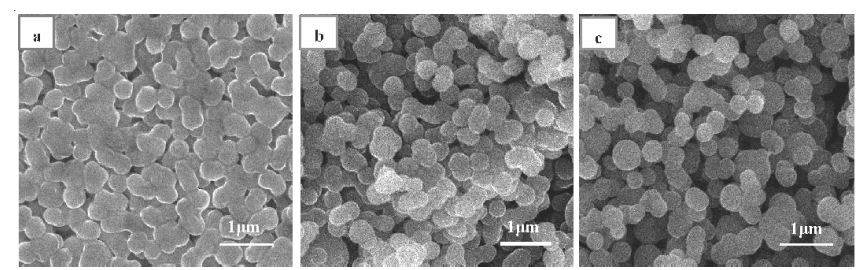

Fig. 1. FESEM images of (a) as-prepared, (b) purified, (c) oxidized-CMSs

\section{Silanization of carbon microspheres}

Influence of reaction time: $\mathrm{KH}-570$ was grafted on the surface of CMSs in different reaction times $(1,2,4,6 \mathrm{~h})$ using $0.3 \mathrm{~g}$ of oxidized CMSs, $1.0 \mathrm{~mL}$ of $\mathrm{KH}-570,45 \mathrm{~mL}$ of ethanol and $15 \mathrm{~mL}$ of water at $65^{\circ} \mathrm{C}$. Fig. 2 shows FESEM images of silanized-CMSs with different reaction times. When the reaction time was $1 \mathrm{~h}$, silanized-CMSs were dispersed in spherical shape with bare surface and little agglomeration owing to the inefficient reaction [Fig. 2(a)]. The silanized CMSs over $2 \mathrm{~h}$ [Fig. 2(b)] showed uniform size and spheric shape. The presence of Si was proved by the EDS spectra on the upper right corner of Fig. 2(b), indicating that KH-570 was grafted on the surface of CMSs. However, with an increase of reaction time to 4 and 6 h [Fig. 2(c), 2(d)], the functionalized CMSs showed more agglomeration.

Influence of KH-570 content: A series of silanized-CMSs with different contents of $\mathrm{KH}-570$ were prepared at $65^{\circ} \mathrm{C}$ for $2 \mathrm{~h}$ with $0.3 \mathrm{~g}$ of oxidized-CMSs and $60 \mathrm{~mL}$ of ethanol + water $(3: 1, v / v)$ (Fig. 3). It can be seen that silanized-CMSs had rough surface with wide and deep grooves. When $3 \mathrm{~mL}$ of KH-570 was added [Fig. 3(a)], the surface of CMSs, on which there were a lot of random polymers, was non-uniform. From its high magnification image [Fig. 3(d)], it is obvious that the coatings on CMSs packed densely and the surfaces of CMSs were very coarse, suggesting that excessive KH-570 was used. As KH-570 content was $2 \mathrm{~mL}$ [Fig. 3(b)], the dispersion of CMSs was improved, but a litter cotton-like coating was still packed on the surfaces of CMSs. With the content of $\mathrm{KH}-570$ decreasing to $1 \mathrm{~mL}$ [Fig. 3(c)], the surfaces of silanized-CMSs [Fig. 3(f)] were covered with more uniform coating layer. With further decreasing KH-570 content to $0.5 \mathrm{~mL}$, the grafting degree obtained by TG analysis was very low because of insufficient KH-570 dosage (not given here). 

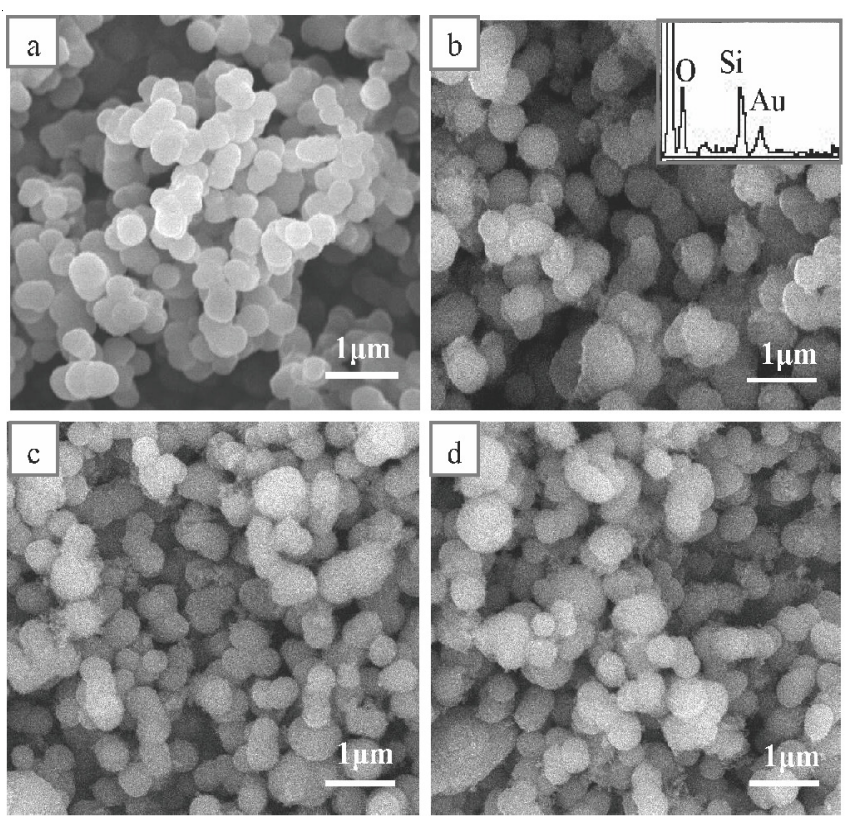

Fig. 2. FESEM images of silanized CMSs in different reaction times: (a) 1 $\mathrm{h}$, (b) $2 \mathrm{~h}$, (c) $4 \mathrm{~h}$, (d) $4 \mathrm{~h}$. Inset on the upper right corner of (b) is the EDS spectra. $(\mathrm{CMSs}=0.3 \mathrm{~g}, \mathrm{KH}-570=1.0 \mathrm{~mL}$, solvent: $45 \mathrm{~mL}$ ethanol $+15 \mathrm{~mL}$ water, $\mathrm{pH} \approx 5, \mathrm{~T}=65^{\circ} \mathrm{C}$ )
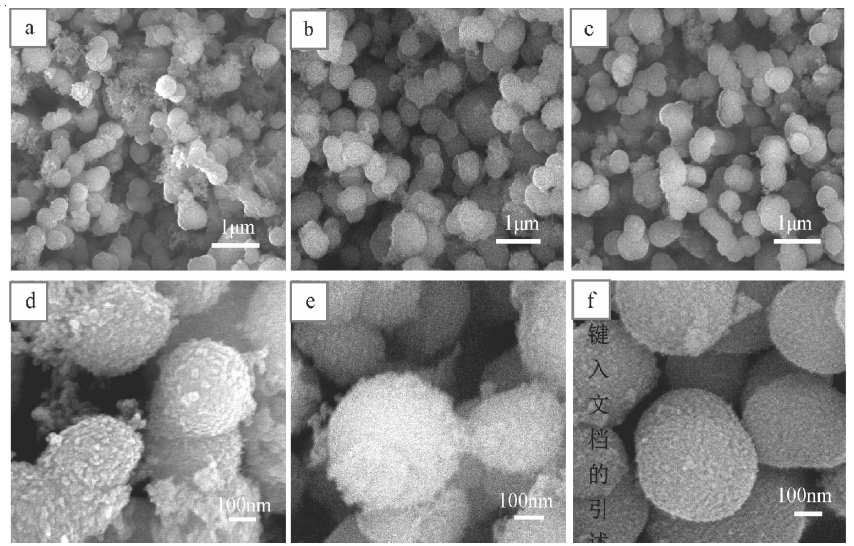

Fig. 3. FESEM images of silanized-CMSs $(0.3 \mathrm{~g})$ with different $\mathrm{KH}-570$ amounts: (a) $3.0 \mathrm{~mL}$, (b) $2.0 \mathrm{~mL}$ and (c) $1.0 \mathrm{~mL}$, (d), (e), (f) are high magnification images of (a), (b), (c), respectively. (CMSs = $0.3 \mathrm{~g}$, solvent: $45 \mathrm{~mL}$ of ethanol $+15 \mathrm{~mL}$ of distilled water, $\mathrm{pH} \approx 5$, $\mathrm{T}=65^{\circ} \mathrm{C}, \mathrm{t}=2 \mathrm{~h}$ )

Influence of the ratio of ethanol to distilled water: Experiments with different ratios of ethanol to water in $60 \mathrm{~mL}$ of co-solvent were carried out to discuss the influence of the solvent. The FESEM images of the products prepared at $65^{\circ} \mathrm{C}$ for $2 \mathrm{~h}$ with $0.3 \mathrm{~g}$ of oxidized-CMSs and $1 \mathrm{~mL}$ of $\mathrm{KH}-570$ are showed in Fig. 4. With pure water as solvent, silanized-CMSs showed many floccules, indicating the self-polymerization among KH-570 molecules [Fig. 4(a)]. As shown in Fig. 4(b) and Fig.4(c), increasing the amount of ethanol improved the self-polymerization of $\mathrm{KH}-570$. When the ratio of ethanol to water reached 3:1, the particles showed uniform size and good dispersion.

Surface functional groups analysis of CMSs: The above results show that the preferable parameters for preparing silanized-CMSs, with $0.3 \mathrm{~g}$ of oxidized-CMSs as raw materials in $60 \mathrm{~mL}$ of ethanol and water at $65^{\circ} \mathrm{C}$, were $1 \mathrm{~mL}$ of $\mathrm{KH}-$ $570,2 \mathrm{~h}$ of reaction time, $3: 1$ of ethanol-to-water ratio.
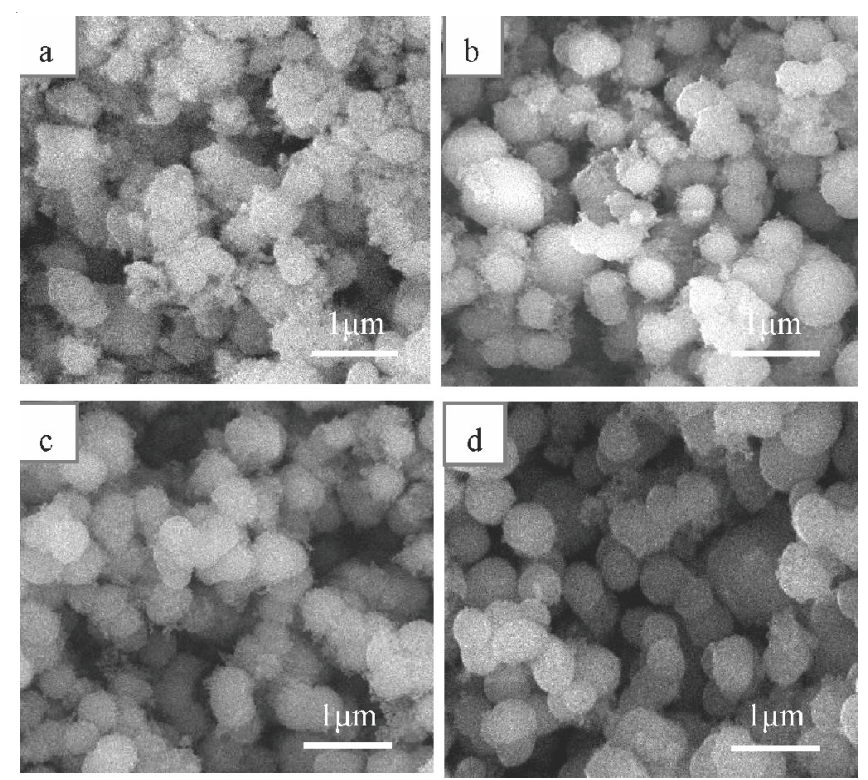

Fig. 4. FESEM images of silanized-CMSs with different ratios of ethanol to water: (a) pure water, (b) $1: 3$, (c) $1: 1$, (d) $3: 1$. (CMSs $=0.3 \mathrm{~g}$, $\mathrm{KH}-570=1.0 \mathrm{~mL}, \mathrm{pH} \approx 5, \mathrm{~T}=65^{\circ} \mathrm{C}, \mathrm{t}=2 \mathrm{~h}$, solvent: $60 \mathrm{~mL}$ )

On the basis of the difference in thermal stability between KH-570 and CMSs, the content of KH-570 grafted onto the surface of CMSs for silanized-CMSs obtained under preferable parameters was measured by TG analysis ${ }^{9}$. Fig. 5 shows the TG curves of the samples. The purified CMSs were stable without evident weight loss up to $500{ }^{\circ} \mathrm{C}$. Oxidized CMSs (curve b in Fig. 5) began to degrade at $300^{\circ} \mathrm{C}$ and had a weight loss of $21.6 \%$ at $800{ }^{\circ} \mathrm{C}$ because of the decomposition and release of oxygen-containing functional groups on their surfaces introduced by mixed acid oxidation ${ }^{3}$. The density of oxygen-containing functional groups was measured by acidbase titration put forward by Boehm et al. ${ }^{12}$. The results indicate that the acidification introduced $3.5699 \mathrm{mmol} / \mathrm{g}$ of oxygencontaining functional groups, including $2.3176 \mathrm{mmol} / \mathrm{g}$ of hydroxyl, $1.2101 \mathrm{mmol} / \mathrm{g}$ of carboxyl and $0.0422 \mathrm{mmol} / \mathrm{g}$ of carbonyl. As shown in Fig. 5(c), the weigh loss of silanized CMSs was $23.2 \%$ between 100 and $800{ }^{\circ} \mathrm{C}$, higher than that of oxidized CMSs. This was attributed to weight loss of KH570 and oxidized CMSs. KH-570 [Fig. 5(d)] appeared to have a weight loss of $38.5 \%$ in the range of $100-800^{\circ} \mathrm{C}$. The grafting percentage of CMSs grafted with $\mathrm{KH}-570$ was calculated as $9.5 \%$ according to eqn. 1 .

Fig. 6 shows the FTIR spectra of purified-CMSs, oxidizedCMSs and silanized-CMSs. For the purified-CMSs [Fig. 6(a)], the bands at 3429 and $1115 \mathrm{~cm}^{-1}$ were attributed to -OH group, which may be from the small amount of adsorbed water. Compared with purified-CMSs, oxidized-CMSs showed new adsorption band at $1593 \mathrm{~cm}^{-1}$ [Fig. 6(b)], which can be identified as the characteristic peaks of the $\mathrm{C}=\mathrm{O}$ stretching vibration. The bands at 3393 and $1090 \mathrm{~cm}^{-1}$ were assigned to the $-\mathrm{OH}$ stretching vibrations. It is obvious that $\mathrm{C}=\mathrm{O}$ and $-\mathrm{OH}$ were introduced on the surface of CMSs after mixed acids oxidation, which is significant for further silanization. Compared with oxidized-CMSs, silanized-CMSs [Fig. 6(c)] shows weak bands at 3432,1568 and $1122 \mathrm{~cm}^{-1}$, suggesting the reaction between $\mathrm{KH}-570$ and $-\mathrm{OH}$ groups of oxidized-CMSs ${ }^{9}$. 


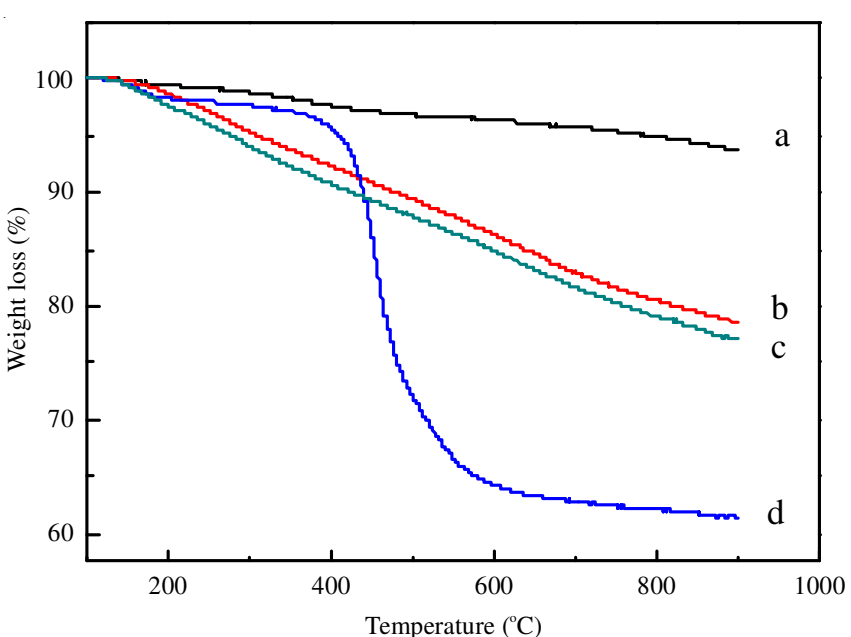

Fig. 5. TG curves of (a) purified, (b) oxidized, (c) silanized CMSs and (d) $\mathrm{KH}-570^{9}$

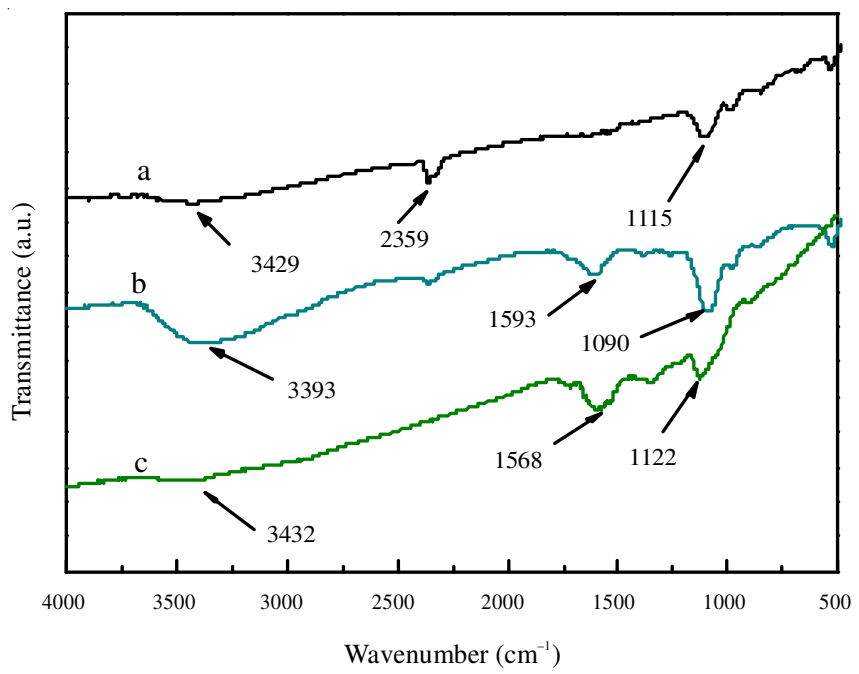

Fig. 6. FT-IR spectra of (a) purified, (b) oxidized, (c) silanized-CMSs

Dispersion in solvent: As shown in Fig. 7, the dispersion of purified, oxidized and silanized CMSs in ethanol was investigated because a mixed solvent of ethanol and distilled water was used in the process of silanization. The samples of CMSs $(0.1 \mathrm{~g}$ for each) were dispersed in $5.0 \mathrm{~mL}$ of ethanol in colorimetric tube separately. After the suspensions were ultrasonicated and then hold still for $24 \mathrm{~h}$. As can be seen, the purified CMSs precipitated, with a smart part floating on the surface of ethanol, suggesting the sample was completely insoluble in ethanol, where as oxidized CMSs remained soluble in ethanol because of introduction of polar functional groups and hydrogen bonding interaction between ethanol and oxygencontaining functional groups on the surfaces of CMSs produced by acid oxidation. The silanized-CMSs were well dispersed in ethanol because of the introduction of organic functional groups onto the surface of CMSs, indicating the dispersion of the silanized-CMSs in ethanol was improved obviously. The silane-functionalized surface of CMSs also possessed high reactivity as a result of the introduction of $-\mathrm{C}=\mathrm{C}$ - bands.

Theoretical analysis of the reaction mechanism: All calculations were carried out within the framework of density functional theory implemented in the DMOL3 package. DMOL3 is a DFT code provided by Accelrys Inc. The generalized
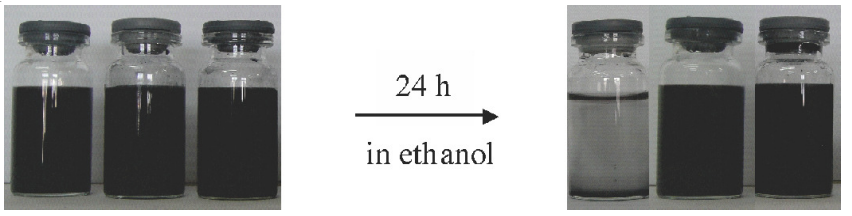

Fig. 7. Dispersion of purified (left), oxidized (middle) and silanized (right) CMSs in ethanol after $24 \mathrm{~h}$

gradient approximation (GGA) with the PBE exchangecorrelation functional and a double numerical basis with polarization functions (DNP) were employed. To include the solvent effect, accurate COSMO method was adopted and the dielectric constant of water was set to be $78.54 \mathrm{~F} / \mathrm{m}$.

Here, we employed cluster model to investigate the chemical reaction between the hydrolyzed KH-570 and the hydroxyl of CMSs surface. Such kind of cluster model was widely used previously ${ }^{13}$. A graphene sheet consisted of $41 \mathrm{C}$ atoms was used to model the CMSs surface and the dangling bonds of the edged $\mathrm{C}$ atom were saturated by $\mathrm{H}$ atom. The graphene sheet was allowed to slightly fold to represent the possible relaxation of the $\mathrm{C}$ atoms on the CMSs surface.

The initial configuration of system is shown in Fig. 8(a). For simplicity, only one hydroxyl group was considered here. In Fig. 8(a), the distance between Si atom in KH-570 and O atom in hydroxyl group is $10 \AA$, implying that the interactions between the molecule and the surface could be ignored. When KH-570 gradually shifts to the hydroxyl group, a transition state (TS) is formed [Fig. 8(b)]. The calculated results show that, in the transition state, the $\mathrm{Si}-\mathrm{O}$ bond length is $2.04 \AA$. The imaginary frequency of the transition state is $-71.3 \mathrm{~cm}^{-1}$ whereas the energy barrier of the transition state is $0.62 \mathrm{eV}$ (equivalent to $59.6 \mathrm{~kJ} / \mathrm{mol}$ ), corresponding to a first-order saddle point on the potential energy surface. Although the theoretical model is quite simple, the low energy barrier of the transition state implies that this reaction could happen under low temperature or high temperature is not necessary. Indeed, as discussed before, the silanization proceeded under $65^{\circ} \mathrm{C}$. Therefore, our theoretical result is in consistence with the experimental observation.

In Fig. 8(c), the products of the reaction are presented. The total energy of the products is $0.34 \mathrm{eV}$ lower than that of the reactants (equivalent to $32.8 \mathrm{~kJ} / \mathrm{mol}$ ), thus the whole reaction is exothermic. In other words, the stability of the surface of CMSs is enhanced after silanization. The strong bonding between KH-570 and surface of CMSs could be qualitatively understood from the deformation charge density, as given in Fig. 8(d). Because only one hydroxyl group was considered here and the theoretical model employed is very simple, more complicated reactions could happen in the actual experiment and these reactions are very difficult to explore by using present cluster model. For example, two adjacent KH-570 may react with each other, forming stable "-Si-O-Si-" reticular bonding. Nevertheless, our theoretical study qualitatively shows that the surface stability is enhanced after silanization. In fact, the enhanced thermal stability of the CMSs surface after silanization was observed in the TG experiment, as can be seen in Fig. 5. Again, the theoretical prediction is verified by experiment. 

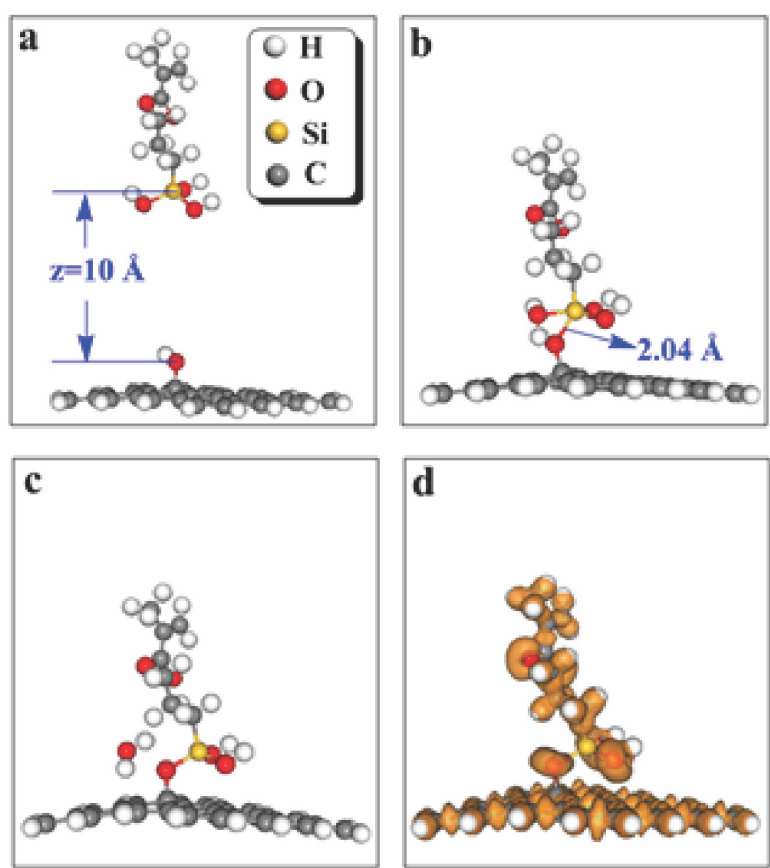

Fig. 8. (a) The initial configuration of system: the hydrolyzed KH-570 and CMSs surface. (b) The transition state of the system. (c) The products of the reaction. (d) The deformation charge density of system under vacuum

Application of surface MIPs in deep desulfurization of fuel oils: On the basis of surface modification of CMSs by $\mathrm{KH}-570$, MIPs on the surface of CMSs (MIP-CMSs) were syhthesized by grafting polymerization using dibenzothiophene (DBT) as template, cholofrom as solvent and 2-arylamide-2methyl propane sulfonic acid (AMPS) as functional monomer 9 For comparing, non-imprinted CMSs (NIP-CMSs) were also prepared in the same way except the addition of DBT. The dynamic adsorption at $25^{\circ} \mathrm{C}$ indicated that when $3 \mathrm{~mL}$ of DBT solution was injected into the packed column, the adsorption of $0.1 \mathrm{~g}$ of MIP-CMSs toward DBT reached saturation with the maximum adsorption amount of $2.54 \mathrm{mg} / \mathrm{g}$, while the same content of NIP-CMSs adsorbed only $0.31 \mathrm{mg} / \mathrm{g}$ of DBT. The imprinting factor reached 8.19 . Generally the specific adsorption of MIPs is signified by the imprinting factor indicated by $\alpha$ : if $\alpha \leq 1$, MIPs do not show selectivity for adsorbate; if 1 $\leq \alpha \leq 1.5$, MIPs exhibit relatively weak selectivity; if $\alpha \geq 1.5$, selectivity is excellent. It is suggested that this kind of MIPCMSs had much better recognition towards DBT than NIPCMSs.

MIP-CMSs for adsorbing DBT molecule were also obtained by in situ polymerization with methacrylic acid (MAA) as the functional monomer ${ }^{1}$. At room temperature by adding $0.1 \mathrm{~g}$ of MIP or NIP-CMSs into $25 \mathrm{~mL}$ of DBT solution in $n$-hexane with initial concentrations of $8 \mathrm{mmol} / \mathrm{L}$, the static adsorption indicated that the adsorption equilibrium time was 5 h. Maximum adsorption capacity of MIP-CMSs (109.5 mg/ g) towards DBT was higher than that of NIP-CMSs $(78.2 \mathrm{mg} /$ g) and the imprinting factor was 1.99, suggesting MIP-CMSs with MAA as monomer had recognition ability and molecular memory towards DBT.

\section{Conclusion}

Carbon microspheres were functionalized by $-\mathrm{C}=\mathrm{C}$ - in $\mathrm{KH}-570$ for further preparation of carbon functional materials. First of all, oxygen-containing polar groups were introduced onto the surface of CMSs by oxidizition. Then $\mathrm{C}=\mathrm{C}$ bond was introduced onto the surface of oxidized CMSs by silanization with coupling agent $\mathrm{KH}-570$. The effects of reaction time, $\mathrm{KH}-$ 570 content and the ratio of ethanol to water on silanization were discussed and the optimum parameters on the basis of $0.3 \mathrm{~g}$ of oxidized CMSs were $1.0 \mathrm{~mL}$ of $\mathrm{KH}-570$, reaction time of $2 \mathrm{~h}$ at $65^{\circ} \mathrm{C}, 3: 1$ of ethanol and water $(60 \mathrm{~mL}), \mathrm{pH} \approx 5$. The grafting degree of CMSs grafted with KH-570 was $9.5 \%$. The dispersibility of silanized-CMSs was improved distinctly in ethanol.

Based on the DFT study, the possible reaction mechanism was discussed. The DFT calculations show that a TS exists when KH-570 reacts with the hydroxyl group on the CMSs surface. Although our cluster model is quite simple, the TS reaction mechanism was suggested for the first time in the present study. Of course, probably more complicated reactions may happen in the actual experiment environments, which will be discussed through more complicated theoretical model in our future research.

\section{ACKNOWLEDGEMENTS}

The authors acknowledged the financial support from Program for Changjiang Scholar and Innovative Research Team in University (IRT0972), National Natural Science Foundation of China (20971094, 21176169, 51152001), International Science \&Technology Cooperation Program of China (2012DFR50460), Natural Science Foundation of Shanxi Province (2009011012-4), Ph. D. Programs Foundation of Ministry of Education of China (20101402110007) and Research Project Supported by Shanxi Scholarship Council of China (2012-038).

\section{REFERENCES}

1. Y.Z. Yang, X.G. Liu, M.C. Guo, S. Li, W.F. Liu and B.S. Xu, Colloid. Surf. A, 377, 379 (2011).

2. Y.Z. Yang, J.J. Song, Y. Li, X.G. Liu and B.S. Xu, J. Mater. Res., 28, 998 (2013).

3. X.M. Guo, Y.Z. Yang and X.G. Liu, Appl. Surf. Sci., 257, 6672 (2011).

4. Y.Z. Yang, X.G. Liu, C.Y. Zhang, M.C. Guo and B.S. Xu, J. Phys. Chem. Solids, 71, 235 (2010).

5. Y.Z. Yang, J.J. Song, Y.X. Han, X.M. Guo, X.G. Liu and B.S. Xu, Appl. Surf. Sci., 257, 7326 (2010).

6. B.S. Xu, J.J. Guo, H.S. Jia, X.W. Yang and X.G. Liu, Catal. Today, 125, 169 (2007).

7. Y.Z. Yang, Y.X. Han, X.G. Liu and B.S. Xu, J. Wuhan Univ. Technol., 27, 454 (2012).

8. Y.J. Xie, A.S. Hill Callum, Z.F. Xiao and H. Militz, Composites A, 7, 806 (2010)

9. Y.Z. Yang, Y. Zhang, S. Li, X.G. Liu and B.S. Xu, Appl. Surf. Sci., 17, 6441 (2012).

10. E.S. Gil and S.M. Hudson, Prog. Polym. Sci., 29, 1173 (2004).

11. H.W. Zhu, J.Q. Wei, K.L. Wang and D.H. Wu, Sol. Energ. Mater. Sol. $C$, 93, 1461 (2009).

12. H.P. Boehm, E. Diehl, W. Heck and R. Sappok, Angew. Chem. Int. Ed., 10, 669 (1964).

13. J.S. Lin, S.Y. Lu and W.C. Chou, Surf. Sci., 605, 131 (2011). 